\title{
A LOCALLY CONNECTED, COMPLETE MOORE SPACE ON WHICH EVERY REAL-VALUED CONTINUOUS FUNCTION IS CONSTANT ${ }^{1}$
}

\author{
J. N. YOUNGLOVE
}

Armentrout [1] has shown that there is a Moore space on which every real-valued continuous function is constant. While this space is connected, it is not known to be complete. In answer to questions raised by $F$. B. Jones [2] it is shown in this paper that there is a connected, locally-connected, complete, separable Moore space on which every continuous real-valued function is constant. This space is very similar to one whose existence was announced by P. Roy [5].

A topological space is called a Moore space if it satisfies Axiom 0 and the first three parts of Axiom 1 of [4]. A Moore space is said to be complete if it satisfies all of Axiom 1 in [4]. In a Moore space, domains are open sets and the set of regions whose existence is assured by Axiom 1 is a base for the topology.

The construction uses a space which is essentially Armentrout's modification of a Moore space constructed by F. B. Jones [3]. It is redescribed here to make the construction more amenable to geometric intuition. The author is indebted to $\mathrm{H}$. Cook for suggesting this geometric realization of the space $\Sigma$.

Let $C$ be a planar disc topologized as follows. If $P$ is on the rim of $C$, regions containing $P$ shall be interiors of circles lying in $C$ and having only $P$ in common with the rim of $C$ together with $P$. If $P$ is in $C$ but not on the rim of $C$, regions containing $P$ shall be interiors of circles lying in $C$ having $P$ in their interior and containing no point of the rim of $C$. With this topology, $C$ becomes a separable, connected, locally connected, complete Moore space $S$. The rim of $C$ is a discrete set in $S$ and is the union of two disjoint uncountable sets $A$ and $B$ with the property that any domain in $S$ which contains an uncountable subset of one of them has an uncountable subset of the other in its closure [3]. Let $M$ be a countable dense subset of $S$ containing no point of the rim of $C$.

Let $T$ be the point set in $E^{3}$ obtained by rotating the graph of $y=x^{2}-1,(-1 \leqq x \leqq 1)$, in the $x y$ plane about the $x$-axis. For each positive integer $n$ let $C_{n}$ be the disc consisting of points in the plane $x=1-1 /(n+1)$ which are interior to or on $T$. For negative integers

Received by the editors December 13, 1966.

1 This research was partially supported by NASA Grant NGR 44-005-037. 
$n$ let $C_{n}$ be the disc consisting of points on the plane $x=-1-1 /(n-1)$ which are interior to or on $T$. Let $C_{0}$ be the disc created by the plane $x=0$ and $T$.

For each integer $n$, the disc $C_{n}$ may be topologized as a copy of $C$ yielding a Moore space $S_{n}$ containing subsets $A_{n}, B_{n}$ and $M_{n}$ which have the same properties with respect to $C_{n}$ and $S_{n}$ that $A, B$ and $M$ have with respect to $C$ and $S$.

The doubly infinite sequence $\cdots S_{-1}, S_{0}, S_{1}, \cdots$ of spaces may be sewn together into a connected space $\Lambda$ by identifying corresponding points of $A_{n-1}$ and $A_{n}$ where $n$ is odd and corresponding points of $B_{n-1}$ and $B_{n}$ where $n$ is even.

Let $\Sigma$ denote the space obtained from $\Lambda$ by adjoining the points $(-1,0,0)$ and $(1,0,0)$ as follows. For each positive integer $n$ the set of all points of all regions containing points of the sets $C_{j}$ for $j \geqq n$ together with the point $(1,0,0)$ shall be a region containing $(1,0,0)$. Regions containing $(-1,0,0)$ are defined similarly.

An extension of Jones' proof in [3] that his space is a Moore space which is not completely regular shows that every real-valued continuous function on $\Sigma$ has the same value at $(-1,0,0)$ as it has at $(1,0,0)$. These two points will be called the "ends" of $\Sigma$.

Let $x_{1}, x_{2}, x_{3}, \cdots$ be a numbering of the points of $M$. This induces a numbering $x_{1 n}, x_{2 n}, x_{3 n}, \cdots$ of points of $M_{n}$ for each integer $n$. Join each $x_{j, n-1}$ to the point $x_{j, n}$ with a copy $\Sigma_{j, n}$ of the space $\Sigma$ by identifying one end of $\Sigma_{j, n}$ with $x_{j, n-1}$ and the other end with $x_{j, n}$. This may be visualized as connecting the points $x_{j, n-1}$ and $x_{j, n}$ by a copy $T_{j n}$ of $T$ lying except for its ends between $C_{n-1}$ and $C_{n}$ and containing a copy $\Sigma_{j n}$ of $\Sigma$ as $T$ contains $\Sigma$. Further, these $T_{j n}$ 's may be placed so that no two intersect except at a common end point and they are all subsets of the interior of $T$. This construction yields a Moore space $\Sigma_{1}$ whose points are the points of all the spaces $\boldsymbol{\Sigma}_{j n}$ subject to the above-mentioned identifications and whose topology is described as follows.

Let $G_{1}, G_{2}, \cdots$ be a sequence of collections of regions in $\Sigma$ satisfying Axiom 1 of [4]. For each $\alpha=(j, n)$ the space $\Sigma_{\alpha}$ is a copy of $\Sigma$ and lias a corresponding sequence $G_{\alpha 1}, G_{\alpha 2}, G_{\alpha 3}, \cdots$ which satisfies Axiom 1. There is a sequence $G_{11}, G_{12}, G_{13}, \cdots$ of collections of connected subsets of $\Sigma_{1}$ such that if "region of $\Sigma_{1}$ " be defined as an element of $G_{11}$, then $\Sigma_{1}$ is a complete Moore space. The regions of each $G_{1 n}$ may be thought of as being of two types. First, every region of $G_{\alpha, n}$ which does not contain an end of $\Sigma_{\alpha}$ is a region of $G_{1 n}$. Second, regions $g$ of $G_{n}$ which are augmented by the addition of points of the space $\left\{\Sigma_{\alpha}\right\}$ as follows. If $g$ contains both end points of some $\Sigma_{\alpha}$ then 
the augmentation of $g$ contains all of $\Sigma_{\alpha}$. If $g$ contains only one end point of a $\Sigma_{\alpha}$ then $g$ is augmented by regions of $G_{\alpha n}$ which contain that end of $\Sigma_{\alpha}$.

The space $\Sigma_{1}$ contains $\Sigma$ as a subspace and has the property that every real-valued continuous function on $\Sigma_{1}$ is constant on $\Sigma$. To see this, consider the sequence of points $\cdots x_{j,-1}, x_{j, 0}, x_{j, 1}, \cdots$ for some fixed $j$. Since these points are ends of the spaces $\Sigma_{j n}, n=0$, $\pm 1, \pm 2, \cdots$ and have the ends of $\Sigma$ as limit points, any real-valued continuous function over $\Sigma_{1}$ is constant over this set. Since the set of all ends of the space $\Sigma_{i j}, i, j=0, \pm 1, \pm 2, \cdots$ is dense in the space $\Sigma$ the space $\Sigma_{1}$ has the property stated above.

By placing copies of $T$ inside each $T_{i j}$ in the same way that $T_{i j}$ 's were placed in $T$, a Moore space $\Sigma_{2}$ may be constructed which contains $\Sigma_{1}$ as a subspace so that every real-valued continuous function $\Sigma_{2}$ is constant on $\Sigma_{1}$. This process may be continued, yielding an infinite sequence $\Sigma_{1}, \Sigma_{2}, \Sigma_{3}, \cdots$ of Moore spaces such that for each positive integer $j, \Sigma_{j+1}$ contains $\Sigma_{j}$ as a subspace and every realvalued continuous function on $\Sigma_{j+1}$ is constant on $\Sigma_{j}$.

In each space $\Sigma_{j}, j>1$, there is a sequence $G_{j 1}, G_{j 2}, \cdots$ of collections of regions obtained from regions of the space $\Sigma_{j-1}$ in the same way that regions for $\Sigma_{1}$ were obtained from regions in $\Sigma$. Note that if $P$ is a point of $\Sigma_{j}$ not in $\Sigma_{j-1}$ then $P$ is not an end point of a copy of $\Sigma$ in $\Sigma_{j}$ and for some integer $k$, regions of $G_{j k}$ which contains $P$ are "between" some two discs in the copy of $\Sigma$ which contains $P$. Further, no future augmentation of such regions will include points not between those two discs.

Let $L$ be the set of all points in spaces in the sequence $\left\{\Sigma_{i}\right\}$, $i=1,2,3, \cdots$ and $K$ be the set of points in $E^{3}$ so that $P \in K$ if and only if there is an infinite nested sequence of copies of $T$ used in the construction of the spaces $\left\{\Sigma_{j}\right\}$ so that $P$ is interior to all the copies of $T$ in this sequence. The set $K \cup L$ may be topologized to form a space $\Sigma_{\infty}$ so that each $\Sigma_{i}$ is a subspace of $\Sigma_{\infty}$ and $\Sigma_{\infty}$ satisfies the requirements listed in the title.

To do this, we first indicate what sets will be regions containing points of $K$. If $P \in K$, then in the construction of $\Sigma_{n+1}$ there was only one copy of $T$ inserted between discs of $\Sigma_{n}$ which contains $P$ in its interior. The set of all points of $K \cup L$ interior to this copy of $T$ will be a region containing $P$. For points $x$ in $L$, we observe that there is a smallest integer $j$ such that $x$ is a point of $\Sigma_{j}$. Let $g$ be a region of $\Sigma_{j}$ containing $P$. There is a sequence $g_{1}, g_{2}, g_{3}, \ldots$ such that for each $m, g_{m+1}$ is a region of $\Sigma_{m+1}$ which was obtained from $g_{m}$ in $\Sigma_{m}$ by an augmentation process as described above. Further, $g_{1}$ is obtained 
from $g$ in this fashion. We create a region of $\Sigma_{\infty}$ containing $P$ by taking the union of the sequence $g_{1}, g_{2}, g_{3}, \ldots$ together with all points of $K$ which are interior to some copy of $T$ used in the construction of a copy of $\Sigma$ which is a subset of some $g_{t}$. From this collection of regions it is possible to create a sequence of collections $H_{1}, H_{2}, H_{3}, \cdots$ of regions of $\Sigma_{\infty}$ which satisfies all of Axiom 1 of [4] and further having the property that each region is a connected set which lies between some two discs of each $\Sigma_{j}$ which it dues not intersect.

The completeness of the space $\Sigma_{\infty}$, i.e. the fourth part of the Axiom 1 of [4] requires the following statement to be true. If $M_{1}$, $M_{2}, M_{3}, \cdots$ is a decreasing sequence of closed sets in $\Sigma_{\infty}$ such that for each positive integer $n$, there is a region $h_{n}$ of $H_{n}$ such that $M_{n} \subset \mathrm{cl} h_{n}$, then there is a point common to all sets of the sequence $\left\{M_{n}\right\}$.

Since each $\Sigma_{j}$ is a complete Moore space in the relative topology created by the sequence $H_{1}, H_{2}, H_{3}, \cdots$ any such sequence $\left\{M_{n}\right\}$ which fails to have a common point must eventually fail to intersect any given $\Sigma_{j}$. This then implies that for some $i, M_{i}$ is a subset of a copy of $T$ used in constructing a copy of $\Sigma$ to build $\Sigma_{j+1}$ from $\Sigma_{j}$. Thus one can follow the sequence $\left\{M_{n}\right\}$ with a nested sequence of copies of $T$ and conclude that some point of $K$ is common to the sequence $\left\{M_{i}\right\}$.

Thus the space $\Sigma_{\infty}$ satisfies the requirements listed in the title.

\section{REFERENCES}

1. Steve Armentrout, $A$ Moore space on which every real-valued continuous function is constant, Proc. Amer. Math. Soc. 12 (1961), 106-109.

2. F. B. Jones, Math. Reviews 22 (1961) \#11365.

3. - Moore spaces and uniform spaces, Proc. Amer. Math. Soc. 9 (1958), 483-486.

4. R. L. Moore, Foundations of point set theory, rev. ed., Amer. Math. Soc. Colloq. Publ. Vol. 13, Amer. Math. Soc., Providence, R. I., 1962.

5. P. Roy, The dual of a Moore space, Notices Amer. Math. Soc. 9 (1962), 327-328.

UNIVERSITY OF HousTON 